Iranian Journal of Pathology | ISSN: 2345-3656

\title{
The Evaluation of Error Types and Turnaround Time of Preanalytical Phase in Biochemistry and Hematology Laboratories
}

\author{
Gokhan Cakirca
}

Dept. of Biochemistry, Sanliurfa Mehmet Akif Inan Training and Research Hospital, Sanliurfa, Turkey

\begin{tabular}{c}
\hline KEYWORDS \\
\hline Preanalytical Error; \\
Specimen Rejection; \\
Quality; \\
Specimen Transportation; \\
Turnaround Time \\
\hline
\end{tabular}

\section{Article Info}

Received 16 Jan 2017; Accepted 05 April 2017; Published Online 17 July 2018;

\section{ABSTRACT}

Background \& objective: Each laboratory should determine the type of errors and turnaround time (TAT), especially in the preanalytical phase to report quality and timeliness of the test results. The current study aimed at investigating the common causes of preanalytical errors in biochemistry and hematology laboratories and evaluating the preanalytical TAT for outpatient samples.

Methods: Data of rejected samples in the laboratory information system from September 2014 to September 2015 were retrospectively reviewed. Also, the preanalytical TAT of the outpatient samples was evaluated over the period of three months from June to August 2015. Preanalytical TAT was calculated from order entry to barcode scanning in the autoanalyzer.

Results: With respect to the ratios of blood sample transfers, 1\% of samples (2305 out of 225,563$)$ in the hematology laboratory and $0.6 \%(1467$ out of 255,943$)$ in the biochemistry laboratory were rejected. The most common cause of rejection in the hematology and biochemistry laboratories was insufficient volume (48.8\%) and hemolyzed sample (74.1\%), respectively. The average preanalytical TAT for the outpatient samples was 62.3 minutes. The preanalytical TAT accounted for $10.8 \%$ (order entry-sample collection), 49\% (sample collection-sample receipt), and 40.2\% (sample receipt-barcode scanning in the autoanalyzer), respectively.

Conclusion: Of all the samples received in the biochemistry and hematology laboratories, the overall percentage of rejections were $0.6 \%$ and $1 \%$, respectively. The main target to improve preanalytical TAT was determined as the transportation (sample collection-sample receipt) step. 


\section{Introduction}

Clinical laboratories play a key role in the diagnosis and treatment of patients since they provide data from the analysis of body fluids. Laboratory errors lead to a number of clinical problems including delayed diagnosis, additional laboratory testing, and incorrect diagnosis and treatment (1). Preanalytical phase errors account for approximately $60 \%-70 \%$ of laboratory errors. Major sources of preanalytical errors include inappropriate test request, patient preparation, specimen collection, specimen transportation, and specimen preparation for testing (2). Reduction of error rates in preanalytical phase is essential to ensure cost effectiveness, patient satisfaction, and quality laboratory service $(3,4)$.

The turnaround time (TAT) used in hospitals to assay the performance of laboratory is defined as the time taken from order entry to result reporting (5). Shortening TAT is important for early diagnosis and treatment that shortens patients' hospital stay and consequently increases their satisfaction and safety $(6,7)$.

The current study aimed at investigating (I) the causes and incidences of the specimens rejection in the preanalytical phase and (II) the preanalytical TAT of outpatient samples for quality and timeliness of reporting the test results.

\section{Materials and Methods}

The current study was conducted at the hematology and biochemistry laboratories of the Mustafa Kemal teaching Hospital. In the current study, the specimens rejected by the hematology and biochemistry laboratories from September 2014 to September 2015 were reviewed. The data were obtained from the laboratory information system (LIS). Furthermore, the preanalytical TAT of outpatient samples of chemistry, immunoassay, coagulation, and hematology test units were evaluated over a period of three months from June to August 2015. The preanalytical phase consists of order entry, barcode printing, sample collection, sample receipt, and barcode scanning in the autoanalyzer steps automatically recorded in LIS as five time points. The time intervals between the stages of preanalytical workflow (order entry-sample collection, sample collection-sample receipt, and sample receiptbarcode scanning in the autoanalyzer) were calculated. The "sample receipt-barcode scanning in the autoanalyzer" step involves distribution of specimens to sections of the lab, centrifugation, and specimen loading.

In the outpatient clinics of the hospital, phlebotomy is usually performed by experienced staff using a vacutainer system. However, in the inpatient services, phlebotomy is mostly performed by nurses and interns using syringes and needles. The blood specimens are transported to the laboratory by the hospital personnel. Then, the specimens are assessed by experienced staff and either accepted or rejected depending on the rejection criteria of the hospital laboratories. The rejection criteria of the laboratory are clotted specimens, insufficient volume, excess volume, specimens with visible hemolysis, lipemic specimens, labelling errors, inappropriate tube, empty tube, and damaged specimens. The current study was approved by the Local Ethics Committee of Mustafa Kemal University.

Statistical analysis was conducted with Microsoft Excel 2010 program. Calculations of rejected specimens for hematology and biochemistry laboratories were presented as number and percentage. Data of preanalytical TAT were expressed as median (min$\max )$.

\section{Results}

Table 1 lists the distribution of hematology and biochemistry samples rejected in the current study. Out of 225,563 samples received in hematology laboratory during the study period, 2305 samples (1\%) were rejected. Out of 255,943 samples received in biochemistry laboratory during the study period, 1467 samples $(0.6 \%)$ were rejected. In the hematology laboratory, the most common cause of rejection was insufficient volume ( $48.8 \%$ of total rejections) followed by clotted specimens $(45.6 \%$ of total rejections). In the biochemistry laboratory, the most common cause of rejection was hemolyzed specimens (74.1\% of total rejections) followed by insufficient volume ( $16.5 \%$ of total rejections). Of the specimens 
175. The Evaluation of Error Types and ...

rejected due to hemolysis, $66 \%$ were collected from the hospital inpatient services and $34 \%$ from the hospital outpatient clinics.

Table 2 shows the analysis of preanalytical TAT of outpatient specimens. The preanalytical TAT for chemistry test units had a median of 62 minutes, immunoassay test units had a median of 73 minutes, coagulation test units had a median of 63 minutes, and hematology test units had a median of 51 minutes.

Table 1. Rejection analysis for hematology and biochemistry specimens

\begin{tabular}{|ccc}
\hline Rejection criteria & $\begin{array}{c}\text { Biochemistry specimen rejection, } \\
\text { frequency; } \mathbf{n}(\mathbf{\%})\end{array}$ & $\begin{array}{c}\text { Hematology specimen rejection, } \\
\text { frequency; n }(\mathbf{\%})\end{array}$ \\
\hline Clotted samples & $32(2.2)$ & $1052(45.6)$ \\
\hline Insufficient samples & $242(16.5)$ & $1124(48.8)$ \\
\hline Excessive amount & - & $45(2)$ \\
\hline Hemolyzed samples & $1087(74.1)$ & $24(1)$ \\
\hline Lipemic samples & $46(3.1)$ & $3(0.1)$ \\
\hline Labelling errors & $33(2.2)$ & $30(1.3)$ \\
\hline Any other reasons ${ }^{\mathbf{a}}$ & $27(1.8)$ & $27(1.2)$ \\
\hline Total Rejection (n) & 1467 & 2305 \\
Total sample (n) & 255,943 & 225,563 \\
\hline
\end{tabular}

a: Inappropriate tube, empty tube and damaged specimens.

Percentages: (Number of rejected samples/Total number of rejected samples) x100.

$\mathrm{b}$ : (The total number of rejected samples/ total number of samples for each laboratory) $\mathrm{x} 100$.

Table 2. Analysis of preanalytical TAT of outpatient specimens

\begin{tabular}{ccccc}
\hline $\begin{array}{c}\text { Laboratory test } \\
\text { groups }\end{array}$ & $\begin{array}{c}\text { Order entry- } \\
\text { Sample collection }\end{array}$ & $\begin{array}{c}\text { Sample collection- } \\
\text { Sample receipt }\end{array}$ & $\begin{array}{c}\text { Sample receipt- } \\
\text { Barcode scanning in } \\
\text { the autoanalyzer }\end{array}$ & $\begin{array}{c}\text { Preanalytical TAT } \\
\text { (minutes) }\end{array}$ \\
\hline $\begin{array}{c}\text { Chemistry } \\
\text { (n: 15,097) }\end{array}$ & $7(1-335)$ & $26(4-276)$ & $29(11-505)$ & 62 \\
$\begin{array}{c}\text { Immunoassay } \\
\text { (n:7,248) }\end{array}$ & $8(1-224)$ & $29(4-236)$ & $36(11-382)$ & 63 \\
$\begin{array}{c}\text { Coagulation } \\
\text { (n:3,094) }\end{array}$ & $6(1-233)$ & $35(4-213)$ & $22(11-299)$ & 51 \\
$\begin{array}{c}\text { Hematology } \\
\text { (n:10,538) }\end{array}$ & $6(1-335)$ & $32(4-276)$ & $13(5-289)$ & 62.3 \\
Average & 6.75 & 30.5 & 25 & $100 \%$ \\
\hline
\end{tabular}

The data were expressed as median (min-max), n: Number of blood samples. Chemistry test units: 30 tests like metabolites, electrolytes, enzymes, lipid profile etc.; immunoassay test units: 27 tests like thyroid function tests, vitamins, fertility hormones, tumor markers etc.; coagulation test units: 5 tests like prothrombine time, partial thromboplastin time, fibrinogen, etc.; hematology test units: 22 tests like hemoglobin, hematocrit, red blood cell count, platelet count, white blood cell count etc.

Table 3. Types of preanalytical errors in the previous studies*

\begin{tabular}{ccccc}
\hline Guimaraes et al. (9) & Sinici Lay et al. (10) & Goswami et al. (11) & Jacobsz et al. (12) & Bhat et al. (13) \\
\hline Clotted & Clotted & Hemolyzed & Clotted & Clotted \\
Insufficient & Insufficient & Insufficient & Insufficient & Labeling errors \\
Hemolyzed & Inappropriate tube/container & Illegible hand writing & Labelling errors & hemolyzed \\
\hline
\end{tabular}

*Types of preanalytical errors are written in order of frequency. 


\section{Discussion}

The reported incidence of the specimens rejected by biochemistry and hematology laboratories ranged $0.3 \%$ to $2.7 \%$ (8-10). Similarly, in the current study, the incidence of the specimens rejected by the biochemistry and hematology laboratories were $0.6 \%$ and $1 \%$, respectively. Table 3 summarizes comparative data regarding types of preanalytical errors in the previous studies (9-13).

In the current study, insufficient volume (48.8\%) was the most common factor leading to specimen rejection in the hematology laboratory and the second common factor in the biochemistry laboratory (45.6\%). Similarly, this factor was the second common cause of specimen rejection in several studies (912). Literature shows that the incidence of insufficient volume is remarkably high in pediatric, neonatal, and oncology wards, in which peripheral vascular access is difficult $(14,15)$. Chawla et al., found that the number of specimens with insufficient volume was higher in the outpatient clinics than inpatient services. The authors suggested that this difference could be attributed to the fact that the phlebotomists working in the blood collection areas perform incorrect phlebotomy practices due to a heavy workload (16). High rates of insufficient volume in the current study could be attributed to the fact that phlebotomy is mostly performed with syringes and needles in the inpatient services of the hospital. This can be due to the point that appropriate and sufficient amount of blood cannot be transferred to specimen collection tubes via syringes and needles, particularly to tubes with anticoagulants.

Clotted specimen was the second most common factor leading to specimen rejection in the hematology laboratory $(45.6 \%)$. In some studies, this factor is the most common factor with an incidence ranging $43.8 \%$ to $55.8 \%(9,10,13)$. Inappropriate handling and pretreatment of blood samples after collection (e g, poor mixing, keeping at horizontal position) is the main reason of the aforesaid clotting problem (17). In accordance with Clinical Laboratory Standards Institute (CLSI) guidelines, it is advised that all blood samples collected in vacuum tubes be mixed gen- tly several times (18). It is believed that paying no attention to this procedure increases the incidence of clotted specimens.

Goswami et al., reported that hemolyzed specimens ( $81 \%$ of total rejections) was the most common cause leading to specimen rejection (11). In the current study, similarly, hemolyzed specimen was the most common cause of rejection in the biochemistry laboratory $(74.1 \%)$. Hemolysis may be caused by a number of conditions including forceful evacuation of a syringe into a tube, prolonged tourniquet application, vigorous mixing of the blood collected into the tube, and the use of inappropriate needles (19). Dorotic et al., found in a questionnaire study that nurses had insufficient knowledge about the reasons of hemolysis (20). The frequency of hemolysis was more in inpatient services than outpatient clinics, similar to the results of the study by Chawla et al. (16). A higher incidence of hemolysis in inpatient services may be due to incorrect phlebotomy techniques and not using vacutainer system for blood collection. Samples in the inpatient services are mostly collected using syringes and needles. One study reported that the phlebotomy technique used for blood collection had a significant effect on the incidence of hemolysis (21). Another study reported that the incidence of preanalytical errors decreased from $61 \%$ to $48 \%$ after using vacutainer system (22).

It is important to reduce turnaround time for early diagnosis and treatment by providing the safety and pleasure of patients. In the current study, preanalytical TAT of outpatient samples was 62.3 minutes and increased preanalytical TAT was primarily due to delayed transportion of samples to the laboratory. Similarly, Kaur et al., found the TAT of preanalytical phase as $50.4 \pm 11.9$ minutes in outpatient department and promotion of sample transportation resulted in prolonged preanalytic TAT (23). In another study, the time taken to preanalytical phase for outpatient chemistry specimens was $29.7 \pm 6.9$ minutes and that was $68.1 \%$ of overall TAT. Additionally, lagging in phlebotomy was the main factor of extended preanalytical stage (24). Delays in transport may result from 
insufficient personnel and the lack of awareness among the hospital staff about the problems arising from delayed transportation. Fernandes et al., reported that using pneumatic tube system to transport blood specimens may shorten the TAT (25). In addii tion, preanalytical phase of turnaround time can be shortened with taking some measurements such as using automatic sample tube barcoding device, choosing appropriate tube, providing sufficient personnel, and educating phlebotomists periodically.

\section{Conclusion}

In the current study, the most frequent causes of specimen rejections were hemolyzed specimen and insufficient volume for biochemistry laboratory. For the hematology laboratory these included clotted specimen and insufficient volume. Also, lagging in transportation was the main factor of prolonged preanalytic TAT. Hence, continuous training should be planned for hospital staff on sample collection techniques (correct techniques for venepuncture, choosing appropriate tube, etc.) and transport time of the specimens.

\section{Conflict of interest}

Authors declared no conflict of interest.

\section{References}

1. Howanitz PJ. Errors in laboratory medicine: practical lessons to improve patient safety. Arch Pathol Lab Med. 2005;129(10):1252-61. PMID: 16196513

2. Lippi G, Chance JJ, Church S, Dazzi P, Fontana R, Giavarina D, et al. Preanalytical quality improvement: from dream to reality. Clin Chem Lab Med. 2011;49(7):1113-26. _https://doi. org/10.1515/CCLM.2011.600 PMID:21517699

3. Sciacovelli L, Plebani M. The IFCC Working Group on laboratory errors and patient safety. Clin Chim Acta. 2009;404(1):79-85. https://doi. org/10.1016/j.cca.2009.03.025 PMID: 19328194

4. Plebani M. Quality indicators to detect pre-analytical errors in laboratory testing. Clin Chim Acta. 2012;33(3):85-8.

5. Hawkins RC. Laboratory Turnaround Time. Clin Biochem Rev. 2007;28(4):179-94.
PMID: 18392122

PMCID:PMC2282400

6. Holland LL, Smith LL, Blick KE. Reducing laboratory turnaround time outliers can reduce emergency department patient length of stay: an 11-hospital study. Am J Clin Pathol. 2005;124(5):672-4. https://doi.org/10.1309/ E9QPVQ6G2FBVMJ3B PMID:16203280

7. Gelrud J, Burroughs H, Koterwas J. Emergency care center turnaround time--an improvement story. J Healthc Qual. 2008;30(1):31-7. PMID: $\underline{18257455}$

8. Dale JC, Novis DA. Outpatient phlebotomy success and reasons for specimen rejection. Arch Pathol Lab Med. 2002;126(4):416-9. PMID: 11900564

9. Guimaraes AC, Wolfart M, Brisolara ML, Dani C. Causes of rejection of blood samples handled in the clinical laboratory of a University Hospital in Porto Alegre. Clin Biochem. 2012;45(1-2):123-6. https://doi.org/10.1016/j. clinbiochem.2011.10.009 PMID:22040813

10. Sinici Lay I, Pinar A, Akbiyik F. Classification of reasons for rejection of biological specimens based on pre-preanalytical processes to identify quality indicators at a university hospital clinical laboratory in Turkey. Clin Biochem. 2014;47(12):1002-5. https://doi.org/10.1016/j. clinbiochem.2014.04.024 PMID:24794787

11. Goswami B, Singh B, Chawla R, Mallika V. Evaluation of errors in a clinical laboratory: a one-year experience. Clin Chem Lab Med. 2010;48(1):63-6. https://doi.org/10.1515/ CCLM.2010.006 PMID:20047530

12. Jacobsz LA, Zemlin AE, Roos MJ, Erasmus RT. Chemistry and haematology sample rejection and clinical impact in a tertiary laboratory in Cape Town. Clin Chem Lab Med. 2011;49(12):2047-50. https://doi.org/10.1515/ CCLM.2011.743 PMID:21995606

13. Bhat V, Tiwari M, Chavan P, Kelkar R. Analysis of laboratory sample rejections in the pre-analytical stage at an oncology center. Clin Chim Acta. 2012;413(15-16):1203-6. https://doi. org/10.1016/j.cca.2012.03.024 PMID:22507083

14. Detaille T, Pirotte T, Veyckemans F. Vascular access in the neonate. Best Pract Res Clin 
Anaesthesiol. 2010;24(3):403-18. https://doi. org/10.1016/j.bpa.2010.02.017 PMID:21033016

15. Gallieni M, Pittiruti M, Biffi R. Vascular access in oncology patients. CA Cancer J Clin. 2008;58(6):323-46. https://doi.org/10.3322/ CA.2008.0015 PMID: 18971486

16. Chawla R, Goswami B, Tayal D, Mallika V. Identification of the types of preanalytical errors in the clinical chemistry laboratory: 1-year study at GB Pant Hospital. Laboratory Medicine. 2010;41(2):89-92. https://doi.org/10.1309/ LM9JXZBMLSVJT9RK

17. Dikmen ZG, Pinar A, Akbiyik F. Specimen rejection in laboratory medicine: Necessary for patient safety? Biochem Med (Zagreb). 2015;25(3):377-85. PMID:26527231 PMCID:PMC 4622196

18. Wayne P. Clinical Laboratory Standards Institute. Collection, Transport, and Processing of Blood Specimens for Testing Plasma-Based Coagulation Assays and Molecular Hemostasis Assays. CLSI H21-A5 document. Clinical Laboratory Standards Institute. 2008.

19. Lippi G, Plebani M, Di Somma S, Cervellin G. Hemolyzed specimens: a major challenge for emergency departments and clinical laboratories. Crit Rev Clin Lab Sci. 2011;48(3):14353. https://doi.org/10.3109/10408363.2011.600228 PMID:21875312 Adrijana Dorotić DA, Vanja Radišić Biljak, Dara
Nedić, Andjelo Beletić. Hemolysis from a nurses' standpoint - survey from four Croatian hospitals. Biochem Med (Zagreb). 2015;25(3):393400. https://doi.org/10.11613/BM.2015.039 PMID:26525069 PMCID:PMC4622201

21. Berg JE, Ahee P, Berg JD. Variation in phlebotomy techniques in emergency medicine and the incidence of haemolysed samples. Ann Clin Biochem. 2011;48(Pt 6):562-5. https://doi. org/10.1258/acb.2011.011099 PMID:21948490

22. Singla P, Parkash AA, Bhattacharjee J. Preanalytical error occurrence rate in clinical chemistry laboratory of a public hospital in India. Clin Lab. 2011;57(9-10):749-52. PMID:22029191

23. Vaneet Kaur KS, Minni Verma, Brinder Chopra. Monitoring of Turnaround time (TAT) in Biochemistry Laboratory of a tertiary care hospital in Punjab. IOSR Journal of Dental and Medical Sciences. 2015;14(10):9-12.

24. Chung HJ, Lee W, Chun S, Park HI, Min WK. Analysis of turnaround time by subdividing three phases for outpatient chemistry specimens. Ann Clin Lab Sci. 2009;39(2):144-9. PMID: 19429800

25. Fernandes CM, Worster A, Eva K, Hill S, McCallum C. Pneumatic tube delivery system for blood samples reduces turnaround times without affecting sample quality. J Emerg Nurs. 2006;32(2):139-43. $\quad$ https://doi.org/10.1016/j. jen.2005.11.013 PMID: $\underline{16580476}$

How to Cite This Article

Cakirca G. The Evaluation of Error Types and Turnaround Time of Preanalytical Phase in Biochemistry and Hematology Laboratories. Iranian Journal of Pathology, 2018; 13(2): 173-178. 